\title{
Net zero healthcare: a call for clinician action
}

\author{
Health professionals are well positioned to effect change by reshaping individual practice, \\ influencing healthcare organisations, and setting clinical standards, argue Jodi Sherman and \\ colleagues
}

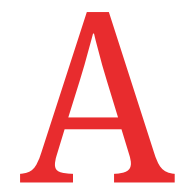

chieving net zero emissions in healthcare will be possible only with radical and immediate engagement of the clinical community. The covid-19 pandemic has served as a wake-up call for high income health systems that resources are finite and globally interdependent, vulnerable to massive surges in demands and simultaneous infrastructure disruption, and that inequities in access to care threaten health and wellbeing for everyone.

During the first months of the pandemic, the medical community united at a historic pace, rapidly sharing information, redesigning models of care, conserving and innovating resources, and moving towards a circular economy. In comparison, the task of transforming healthcare culture and practice to halve healthcare emissions by 2030 as recommended by the Intergovernmental Panel on Climate Change $^{1}$ seems entirely feasible.

\section{Planetary healthcare}

Planetary healthcare requires embracing an expanded notion of the principle "first do no harm," beyond care for individual patients to a duty to protect the Earth's natural systems on which intergenerational

\section{KEY RECOMMENDATIONS}

- Clinicians must work to reduce the incidence and severity of disease to decrease the amount and intensity of care required

- Use of resources must be optimised by ensuring appropriate care and avoiding unnecessary investigations and treatments

- Coordination of care between different providers should be promoted to avoid duplication of services and reduce travel emissions and unnecessary building use

- Health professionals should encourage change through individual practice, influencing healthcare organisations, and contributing to standards and policy health and wellbeing depend. ${ }^{2}$ This planetary health lens acknowledges crucial links between ecological change, human health, and our ability to thrive. ${ }^{2}$

Planetary accountability encompasses actions taken by individual health professionals within the clinical setting, collective actions of clinicians in healthcare organisations with the communities they serve, and interactions of individuals and collectives in professional societies with regulatory and oversight bodies.

For clinicians, this means recognising that healthcare consumes finite resources and produces harmful pollution, accepting that environmental stewardship is integral to our fundamental duty of care, and that we are quickly approaching a climate tipping point.

Healthcare is one of the largest polluting industries, responsible for nearly $5 \%$ of total global greenhouse gases. ${ }^{3}$ Like all industries, healthcare must rapidly and substantially reduce its greenhouse gas emissions to avoid the most catastrophic outcomes to health and wellbeing from climate change.

Achieving net zero emissions-that is, reducing carbon output until it is in balance with natural and engineered means of absorption-necessitates optimising the efficiency and environmental performance of healthcare delivery. However, these alone are insufficient. We must also work to reduce the incidence and severity of disease to decrease the amount and intensity of care required. Furthermore, we must match supply of health services to their need, by ensuring appropriate care and avoiding unnecessary investigations and treatments. In this way, absolute emissions can be reduced while expanding access to healthcare and achieving co-benefits from mitigating harm and costs from healthcare pollution.

Health professionals are well positioned agents of change at many levels, from shaping individual clinical practices to influencing healthcare organisations and setting standards and policy. We have previously published a planetary healthcare framework setting out three strands of action: reducing emissions from healthcare services, matching supply and demand, and reducing demand for healthcare. ${ }^{4}$ Here we provide practical suggestions to help clinicians enact that framework (table 1).

\section{Reducing emissions from supply of health services}

Reducing emissions from healthcare services encompasses all activities that consume materials and energy. Most healthcare sustainability initiatives focus on large scale facility operations, such as improving hospital energy performance and sourcing renewable electricity, which typically are not under the control of clinicians. However, clinicians influence building use through decisions on care settings-for example, whether to administer monitoring or treatment in the home, clinic, or hospital (which has the highest resource and emissions intensity). ${ }^{5}$ Virtual care for patient-provider interactions that do not require in-person examination reduces travel and clinic emissions, obviating the need for some clinical spaces, as seen in the covid-19 pandemic.

Coordination between care providers, such as through arranging multidisciplinary consultations and services on the same day, and proximal diagnostic testing, can further minimise emissions from patient travel. Such changes often require reorganisation of processes and commitment, which can be hindered by lack of understanding of the need for coordination.

The majority of health sector emissions are embedded in the supply chain, including pharmaceuticals and medical devices. ${ }^{5}$ Embedded emissions are dictated by materials and design, as well as production and distribution practices. Use of organisational purchasing power and regulatory reform to influence manufacturers to reduce product emissions is critical but takes time. Clinicians have an immediate role through preferential use of lower emissions supplies (such as choosing reusable rather than single use medical devices, ${ }^{6}$ and dry powder 


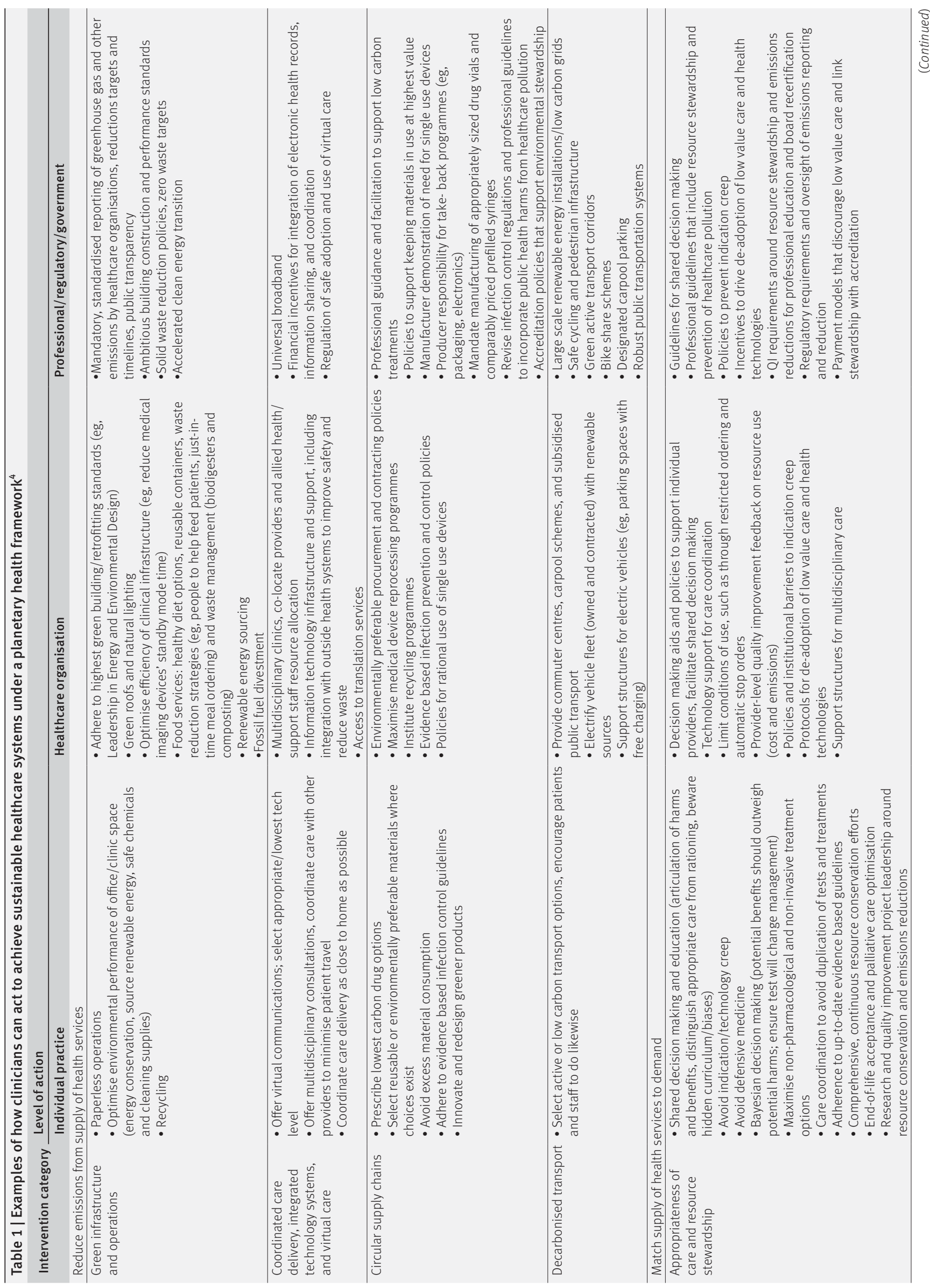

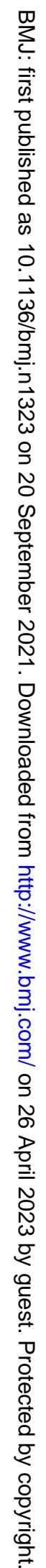




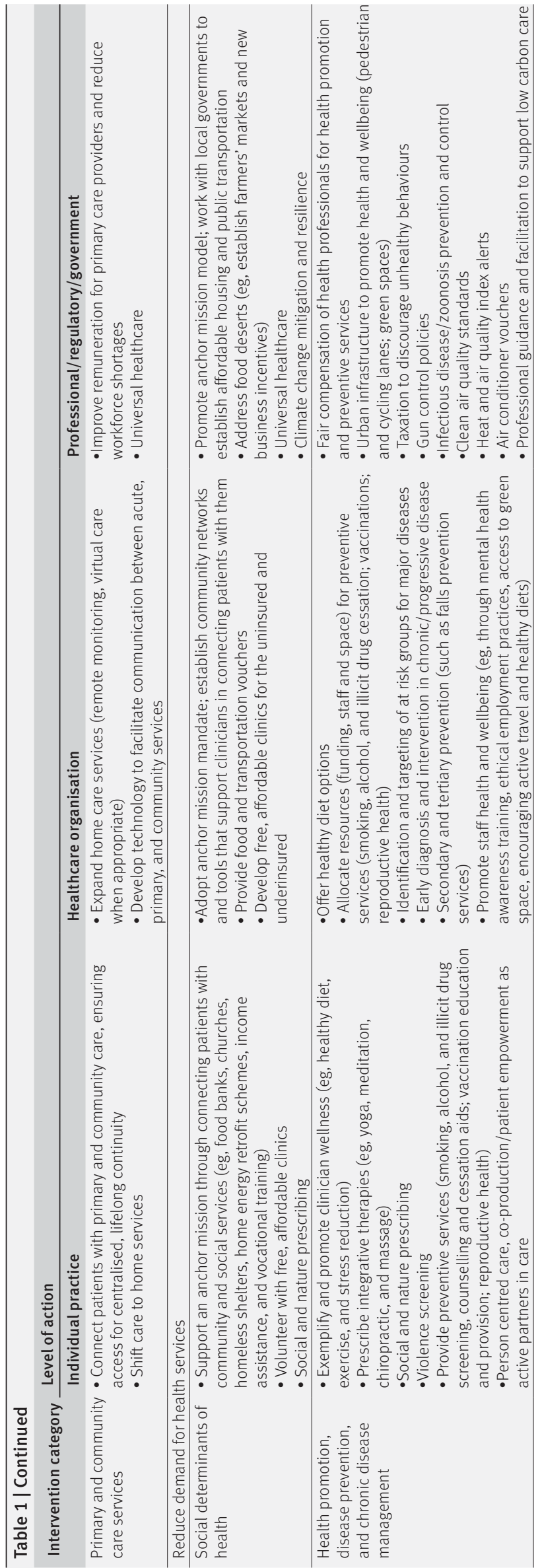

inhalers over metered dose inhalers), and especially through reducing unnecessary consumption of supplies and treatments in their clinical practice.

Matching supply to demand of health services Inappropriate or low value care, in which harms or costs outweigh benefits, is ubiquitous in health systems in both high and low income settings. It includes overuse and underuse of healthcare services, which often coexist in the same health system (and even for the same patient). Mismatches between supply and demand of health services occur because of health system structure and funding and behaviours of clinicians and patients that drive misuse.

Underuse of necessary services leaves patients vulnerable to avoidable disease. Overuse results in harms to patients from adverse events and exposures, financial harms to health systems and possible supply shortages, and population level disease burden from pollution generated by healthcare. Appropriate care optimises health and wellbeing by delivery of what is needed, wanted, clinically effective, affordable, equitable, and responsible in its use of resources. ${ }^{7}$ High value care also maximises environmental performance, avoiding harm to public health.

A robust primary care system is foundational to appropriate care and provides a platform for overcoming barriers to change. ${ }^{8}$ In high income countries, lack of access to, or inadequate primary and preventive care services results in patients interacting with more resource intensive health services such as hospital based treatment. For example, patients may present with advanced disease that would have been preventable or manageable if detected earlier.

Clinicians can mitigate unnecessary use of hospital services by facilitating access to primary and community care services. This includes identifying and targeting underserved groups, moving beyond treating the results of ethnic and economic disparities and seeking to tackle the root cause of inequities by building community wealth (the "anchor mission"). Screening patients for the social determinants of health can identify those at risk and guide health systems to influence community investments. Clinicians can also engage in innovative delivery models that allow care historically offered in the acute setting to be delivered in the community (for example, using remote physiological monitoring and mobile apps.) 
In light of the many harms resulting from inappropriate delivery of health services, clinical decision making should be viewed through a stewardship lens-that is, the careful and responsible management of healthcare resources entrusted to providers. Instead, evidence indicates widespread overuse of resources such as medical supplies, medications (beyond opioids and antibiotics), ${ }^{9}$ and laboratory and radiological investigations. ${ }^{10}$

Globally, a quarter of the total volume of healthcare services is low value. ${ }^{7}$ Solutions include clinician education and empowerment, development of and adherence to evidence based standards of care that incorporate environmental harms, de-adoption ${ }^{11}$ of low value care, shared decision making, care coordination, and continuous quality improvement, all grounded in a fundamental duty of resource stewardship and care for planetary health.

\section{Evidence and education}

Formal education should include training in planetary health and stewardship principles. ${ }^{12}$ Continuing education is required to remain up to date on best practices, as well as indications for specific tests and interventions. The ability to critically appraise evidence, extrapolate findings to appropriate patient populations, and identify industry influence or conflicts of interest is essential to providing high value care.

By keeping their knowledge thorough and current, health professionals can protect against "technology creep"-the application of technologies or treatments to expanded indications without supporting evidence. New evidence or alternative technologies can also result in existing technologies or practices becoming inappropriate or obsolete, necessitating de-adoption strategies. ${ }^{11} \mathrm{~A}$ core driver of resource misuse is ignorance of the evidence and failure to change practice. ${ }^{13}$ This is compounded by ethical failures around resource stewardship and lack of appreciation of the rapid rate of environmental degradation and healthcare's contribution to it.

It is also important to understand the risks and benefits of different options, including non-pharmacological and noninvasive approaches. This knowledge can help patients to have appropriate expectations of what is knowable and treatable. Rather than striving for "zero harm," which is unattainable and results in unintended consequences, clinicians should embrace risk reduction. ${ }^{14} \mathrm{~A}$ risk reduction approach considers implications for both the individual patient and society, including from consumption of finite resources and pollution generation.

Health professionals must apply current evidence, critically evaluating the likelihood that results of available tests will inform management decisions or that treatments will achieve desired outcomes. If early detection has no benefit, patients should be spared the inconvenience and anxiety of close screening or surveillance and the potential harm from treating false positive findings. Effective communication is essential to dispel mistaken notions that resource stewardship is synonymous with withholding care.

\section{Shared decision making}

Shared decision making involves clinicians helping patients incorporate personal values and preferences into the weighing of risks and benefits to arrive at tailored solutions that best meet their needs. This requires an appreciation of the harms of overdiagnosis and overmedicalisation. Shared decision making embraces a biopsychosocial approach to care and honours patient goals, tending to result in less inappropriate disease focused treatment (for example, chemotherapy at end of life, and stenting in stable coronary artery disease).$^{13}$ Studies of shared decision making aids have shown that $20 \%$ of elective procedures would be unwanted if patients had access to understandable, relevant clinical information. ${ }^{13}$

\section{Care coordination}

Inadequate communication and coordination between providers lead to duplicated and unnecessary services because of incomplete information about a patient's history and current circumstances. Seamless and adequate communication between primary care providers and specialists, and between specialty services such as in multidisciplinary cancer teams, avoids unnecessary care, improves safety, and provides a better patient experience. Barriers to this coordination can be reduced by dedicated staff and supporting technology such as shared access to electronic health records among different healthcare organisation networks and non-affiliated practices.

\section{Institutional structures to drive high value} care

Clinicians can work with their healthcare organisations to develop and implement structures that promote adherence to evidence based best practices and discourage wasteful practices. Restrictions on antibiotic and opioid ordering, ${ }^{15}$ automatic stop dates on laboratory investigations, and alerts for high fresh gas flow during anaesthesia embed stewardship into electronic health records.

Institutional policies-for example, those that recommend against routine prophylaxis for stress ulcers (which data show is harmful ${ }^{16}$ ) or restrict access to desflurane (because of its disproportionate climate impact ${ }^{17}$ ), hasten the uptake of knowledge of harms and facilitate de-adoption of low value care. Specialist teams can standardise aspects of inpatient care and ensure up-to-date best practice through electronic decision support and benchmarking tools.

Developing clinical practice guidelines through professional societies lessens the responsibility on individual clinicians and confers a degree of medicolegal protection. Similarly, hospital policies and procedures can diffuse decision making responsibility, removing pressure that drives clinicians to practise defensive medicine or relieving ethical dilemmas around appropriate allocation of limited resources and endof-life care, as happened in the covid-19 pandemic. $^{18}$

\section{Continuous quality improvement}

Environmental performance should be integrated into the core definition of quality care, with best practices established for clinicians and health systems and reinforced through regulatory and oversight processes that overcome obstacles to change. Investigations of appropriateness of care and resource consumption lend themselves to quality improvement initiatives, which can be designed, initiated, and carried out by individual clinicians within their professional settings. Electronic health records can provide feedback to clinicians on resource use, costs, and emissions, to gauge performance and drive quality improvement. ${ }^{19}$

\section{Reducing demand for health services}

Reducing demand for health services requires tackling drivers of poor health. In the United States, over $50 \%$ of healthcare services are devoted to the $5 \%$ of the population with advanced chronic disease. ${ }^{20}$ Most advanced disease develops in people who had risk factors or early stages of illness that were preventable or reversible, often through behavioural and lifestyle approaches alone. ${ }^{21}$ Furthermore, healthcare services contribute to only $20 \%$ of health and wellbeing, with the remain- 
der being the result of broader social, economic, ecological, and political factors. ${ }^{22}$ However, current healthcare strategies routinely neglect social determinants of health, missing opportunities to reduce the burden, expense, and environmental effect of chronic disease. An integrative healthcare framework offers a potential solution. $^{23}$

Integrative healthcare is the delivery of non-pharmacological and lifestyle approaches to disease prevention and treatment in coordination with conventional treatments of chronic disease. ${ }^{24}$ Smoking cessation, reducing use of drugs (including alcohol), and better dietary habits, activity levels, and stress management can prevent or mitigate many chronic diseases. ${ }^{7}$ Evidence based approaches such as yoga, acupuncture, massage, and mind-body practices are particularly useful for pain reduction and more appropriate than medications (especially opioids) for chronic pain. ${ }^{25}$ As part of primary care, these approaches offer opportunities to intervene upstream in health promotion and disease prevention. ${ }^{23}$ While these behavioural and social determinants are not the sole responsibility of healthcare services, helping patients better engage and manage them could go a long way towards reducing the need for more expensive and environmentally damaging interventions later.

Prescribing nature based interventions and activities such as local walking groups, community gardening, and food growing projects can help meet health needs. Benefits of green time are most researched in mental health, with protected areas worldwide estimated to be worth the equivalent of $\$ 6 \mathrm{tn}$ ( $£ 4 \mathrm{tn}$; $€ 5 \mathrm{tn})$ annually in mental health services. ${ }^{26}$ Recommending patients engage socially in local community services can help tackle some of the social determinants of health such as food insecurity and social isolation.

\section{Closing the information and practice gap}

Environmental engineering tools and methods to quantify carbon and other environmental emissions are well established, and life cycle assessment is the gold standard in healthcare sustainability research. ${ }^{27}$ Although the emissions and public health damages from low value care are not yet known, it stands to reason that reducing unnecessary care would reduce emissions and costs, provided that the emissions intensity of required care is simultaneously reduced.
The process of mobilising the clinical community around planetary healthcare requires a concomitant investment in knowledge generation to identify environmentally preferable practices, establish evidence around high value care, and guide public policy for optimal population health. Clinicians should take the lead in advancing this research agenda, while healthcare institutions, universities, and funding bodies must support the work by prioritising planetary health mandates and providing appropriate resources.

Competing interests: We have read and understood BMJ policy on declaration of interests and have no interests to declare.

Provenance and peer review: Commissioned; externally peer reviewed.

This article is part of a series commissioned by The BMJ for the World Innovation Summit for Health (WISH). The BMJ peer reviewed, edited, and made the decisions to publish. The series, including open access fees, is funded by WISH.

Jodi D Sherman, associate professor ${ }^{1}$

Forbes McGain, consultant ${ }^{2,3,4}$

Melissa Lem, clinical assistant professor ${ }^{5}$

Frances Mortimer, medical director ${ }^{6}$

Wayne B Jonas, clinical professor $r^{7,8,9}$

Andrea J MacNeill, clinical associate professor ${ }^{10}$

${ }^{1}$ Department of Anesthesiology, Yale School of Medicine, Connecticut, USA

${ }^{2}$ Western Health, Footscray, Melbourne, Australia

${ }^{3}$ Department of Critical Care, University of Melbourne, Melbourne, Australia

${ }^{4}$ School of Public Health, University of Sydney, Sydney, Australia

${ }^{5}$ Department of Family Practice, University of British Columbia, Vancouver, Canada

${ }^{6}$ Centre for Sustainable Healthcare, Oxford, England ${ }^{7}$ Samueli Integrative Health Programs, Corona Del Mar, CA, USA

${ }^{8}$ Georgetown University School of Medicine, Washington, DC, USA

${ }^{9}$ Uniformed Services University, Maryland, USA

${ }^{10}$ Department of Surgery, University of British Columbia, Vancouver, Canada

Correspondence to: JDSherman

jodi.sherman@yale.edu

\section{(c) (1) (8)}

This is an Open Access article distributed in accordance with the Creative Commons Attribution Non Commercial (CC BY-NC 4.0) license, which permits others to distribute, remix, adapt, build upon this work non-commercially, and license their derivative works on different terms, provided the original work is properly cited and the use is non-commercial. See: http://creativecommons.org/ licenses/by-nc/4.0/.

\section{Check for updates}

1 Intergovernmental Panel on Climate Change. Global warming of $1.5^{\circ} \mathrm{C}$. An IPCC special report. 2018. https://www.ipcc.ch/sr15/.
Wabnitz KJ, Gabrysch S, Guinto R, et al. A pledge for planetary health to unite health professionals in the Anthropocene. Lancet 2020;396:1471-3. doi:10.1016/S0140-6736(20)32039-0

3 Watts N, Amann M, Arnell N, et al. The 2020 report of the Lancet Countdown on health and climate change: responding to converging crises Lancet 2021;397:129-70. doi:10.1016/S01406736(20)32290-X

4 MacNeill AJ, McGain F, Sherman JD. Planetary health care: a framework for sustainable health systems. Lancet Planet Health 2021;5:e66-8. doi:10.1016/ S2542-5196(21)00005-X

5 Eckelman MJ, Sherman J. Environmental impacts of the US health care system and effects on public health. PLoS One 2016;11:e0157014. doi:10.1371/journal.pone.0157014

6 MacNeill AJ, Hopf H, Khanuja A, et al. Transforming the medical device industry: road map to a circular economy. Health Aff (Millwood) 2020;39:2088-97. doi:10.1377/hlthaff.2020.01118

7 Lown Institute. Right care series in the Lancet. 2017. https://lowninstitute.org/projects/right-care-seriesin-the-lancet/.

8 Elshaug AG, Rosenthal MB, Lavis IN, et al. Levers for addressing medical underuse and overuse: achieving high-value health care. Lancet 2017;390:191-202. doi:10.1016/S0140-6736(16)32586-7

9 Fleming-Dutra KE, Hersh AL, Shapiro DJ, et al. Prevalence of inappropriate antibiotic prescriptions among US ambulatory care visits, 2010-2011. JAMA 2016;315:1864-73. doi:10.1001/ jama.2016.4151

10 Hendee WR, Becker GJ, Borgstede JP, et al. Addressing overutilization in medical imaging. Radiology 2010;257:240-5. doi:10.1148/radiol.10100063

11 Powers BW, Jain SH, Shrank WH. De-adopting lowvalue care: evidence, eminence, and economics. JAMA 2020;324:1603-4. doi:10.1001/jama.2020.17534

12 Omrani OE, Dafallah A, Paniello Castillo B, et al. Envisioning planetary health in every medical curriculum: An international medical student organization's perspective. Med Teach 2020;42:1107 11. doi:10.1080/0142159X.2020.1796949

13 Saini V, Garcia-Armesto S, Klemperer D, et al. Drivers of poor medical care. Lancet 2017;390:178-90. doi:10.1016/S0140-6736(16)30947-3

14 Amalberti R, Vincent C. Managing risk in hazardous conditions: improvisation is not enough. BMJ Qual Saf 2020;29:60-3. doi:10.1136/ bmjqs-2019-009443

15 Pollack LA, Srinivasan A. Core elements of hospital antibiotic stewardship programs from the Centers for Disease Control and Prevention. Clin Infect Dis 2014;59(Suppl 3):S97-100. doi:10.1093/cid/ciu542

16 Grady D, Redberg RF. Less is more: how less health care can result in better health. Arch Intern Med 2010;170:749-50. doi:10.1001/ archinternmed.2010.90

17 Zuegge KL, Bunsen SK, Volz LM, et al. Provider education and vaporizer labeling lead to reduced anesthetic agent purchasing with cost savings and reduced greenhouse gas emissions. Anesth Analg 2019;128:e97-9. doi:10.1213/ ANE.0000000000003771

18 Emanuel EJ, Persad G, Upshur R, et al. Fair allocation of scarce medical resources in the time of covid-19. N Engl J Med 2020;382:2049-55. doi:10.1056/ NEJMsb2005114

19 Sherman JD, MacNeill A, Thiel C. Reducing pollution from the health care industry. JAMA 2019;322:10434. doi:10.1001/jama.2019.10823

20 Waters H, Marlon G. The costs of chronic disease in the US. Milken Institute, 2020. https:// milkeninstitute.org/reports/costs-chronic-disease-us.

21 McGinnis JM, Williams-Russo P, Knickman JR. The case for more active policy attention to health promotion. Health Aff (Millwood) 2002;21:78-93. doi:10.1377/hlthaff.21.2.78 
22 University of Wisconsin Population Health Institute. County health rankings and roadmaps: building a culture of health county by county. https://www. countyhealthrankings.org L

23 Jonas WB. A new model of care to return holism to family medicine. I Fam Pract 2020:69:493-8.

24 Egger G, Dixon J. Beyond obesity and lifestyle: a review of 21 st century chronic disease determinants. Biomed Res Int 2014;2014:731685. doi:10.1155/2014/731685
25 Qaseem A, McLean RM, O'Gurek D, Batur P, Lin K, Kansagara DL. Nonpharmacologic and pharmacologic management of acute pain from nonlow back, musculoskeletal injuries in adults: a clinical guideline from the American College of Physicians and American Academy of Family Physicians. Ann Intern Med 2020;173:739-48. doi:10.7326/M193602

26 Buckley R, Brough P, Hague L, et al. Economic value of protected areas via visitor mental health. Nat
Commun 2019;10:5005. doi:10.1038/s41467019-12631-6

27 Sherman JD, Thiel C, MacNeill A, et al. The green print: advancement of environmental sustainability in healthcare. Resour Conserv Recycling 2020;161:104882. doi:10.1016/j. resconrec.2020.104882

Cite this as: $B M / 2021 ; 374: n 1323$

http://dx.doi.org/10.1136/bmj.n1323 